\title{
A Case Control Study of Procalcitonin as A Novel Bio Marker in Sepsis
}

\author{
Dr. B.Preethi ${ }^{1}$, Dr. M. Roopalatha ${ }^{2}$, Dr. C.Ramakrishna ${ }^{3}$, Dr. S.Sanjeevi Rao ${ }^{4}$, \\ B.Venkateswara Rao ${ }^{5}$. \\ NRI Medical College, Sangivalsa. Visakhapatnam.
}

\begin{abstract}
Background: Sepsis is a leading cause of mortality in critically ill patients .Early diagnosis of sepsis followed by appropriate treatment decreases mortality and morbidity.The aim of this study is to assess the role of procalcitonin as a marker in the early diagnosis of sepsis.

Methods: A total of 50 patients with sepsis admitted to AMCU of seven hills hospital, Visakhapatnam from 111-2015 to 1-11-2016 were included in the study.Another 50 healthy persons with no clinical or biological data of infection, age and sex matched were included as a control group.Subjects were subjected to a thorough history taking and routine laboratory investigations.Serum procalcitonin was measured using Elecys PCT assay on Roche Cobas e411 analyser.W.B.C was estimated by Sysmex xs -1000i Hematology Analyser.

Results:Mean levels of PCT and WBC in cases were significantly higher than in control groups, $p<0.001$. when compared PCT showed a direct positive correlation with WBC.
\end{abstract}

Conclusion:PCT alone or combined with WBC is a useful biomarker in early diagnosis of sepsis.

Keywords: Procalcitonin,Sepsis.

\section{Introduction}

Animals mount both local and systemic responses to microbes that traverse their epithelial barriers and enter underlying tissues.Fever or hypothermia,leukocytosis or lecopenia, tachypnea and tachycardia are the cardinal signs of systemic response that is often called as the systemic inflammatory response syndrome(SIRS).SIRS may have a infectious or a non infectious etiology.If a infection is suspected or proven a patient with SIRS is said to have sepsis. When sepsis is associated with dysfunction of organs distant from site of infection the patient has severe sepsis.Severe sepsis may be accompanied by hypotension or evidence of hypoperfusion. When hypotension cannot be corrected by infusing fluids the diagnosis is septic shock.(1) Sepsis is a potentially life threatening condition in which there is a widespread inflammatory state caused by the release of inflammatory mediators,including cytokines and kinins. These inflammatory mediators are released in response to infection and cause damage to the endothelium of blood vessels which allow them to leak fluid.This causes tissue edema ,hypotension and hypoperfusion of organs.It also activates the clotting cascade, which leads to disseminated intravascular coagulation(DIC). The hypoperfusion of organs from hypotension or DIC may result in multiple organ failure and death.(2)

Procalcitonin is a 116 aminoacid peptide that has approximate molecular weight of $14.5 \mathrm{kda}$ and belongs to the calcitonin (CT)super family of peptides(3).Procalcitonin is encoded by calc -1 gene located on chromosome 11.Since the mid 1990's there has been an increasing use of PCT measured in identifying sysmetic bacterial infections.(4)The short half life (25-30hours in plasma)of PCT,coupled with its virtual absence in health and specificity for bacterial infections gives it a clear advantage over the other markers of bacterial infections $(3,4)$.

\section{Materials And Methods}

This prospective case -control study was conducted in seven hills hospital in visakhapatnam from 111-2015 to 1-11-2016.The study was approved by institutional ethical committee.The study sample included all patients aged above 18years presenting consecutively to our centre during the study period with acute sepsis,as diagnosed by one of the following; Clinical presentation of sepsis with positive blood culture,clinical presentation of urinary tract infection with positive urine culture,clinical presentation of pneumonia with supporting radiological features and positive sputum culture or other conditions with clinical and laboratory features compatible with sepsis

Patients with history of malignancy ,trauma or recent surgery were excluded from the stud Blood samples were drawn from all subjects within 24hrs of admission to the AMCU for complete blood count,procalcitonin, blood culture and other related tests.Serum PCT was measured using Elecys PCT assay on Roche Cobas e411 analyser. The Elecys PCT assay is a two step sandwich immunoassay with steptavidin 
microparticles and an electro chemiluminiscence detection system. The test system reagents contain a biotinylated monoclonal PCT specific antibody and a ruthenium labeled monoclonal specific antibody.Total duration of assay $-18 \mathrm{~min} .1^{\text {st }}$ incubation:Antigen in sample $(30 \mu \mathrm{l})$, a biotinylated monoclonal pct specific antibody labeled with a ruthenium complex react to form a sandwich complex. $2^{\text {nd }}$ incubation:After addition of steptavidin coated microparticles the complex becomes bound to the solid phase via interaction of biotin and steptavidin.

The reaction mixture is aspirated into the measuring cell where the microparticles are magnetically captured onto the surface of the electrode .Unbound substances are then removed with procell/procellm. Application of a voltage to the electrode then induces chemilumininscent emission which is measured by a photomultiplier.Results are determined via a calibration curve which is instrument specifically generated by a 2 point calibration and a master curve provided via the reagent barcode. The measuring range claim for the Elecys PCT assay is $0.02-100 \mathrm{ng} / \mathrm{ml}$.WBC count was estimated by Sysmex,xs-1000i Haematology Analyser.Estimation was done using fluorescence flow cytometry technology.

\section{Statistical analysis}

Statistical analysis was done using chi-square analysis and student ' $t$ ' test.p value $<0.05$ is significant

\section{Results}

Overall 50 patients and 50 controls were included in the study.Figure 1 shows mean and SD of PCT in cases and controls.Figure 2 shows mean and SD of WBC in cases and controls.Figure 3 shows correlation of PCT with WBC in cases.

Table No 1. Comparison of PCT Values in Cases \& Controls: $\left(\mathrm{n}_{1}=50, \mathrm{n}_{2}=50\right)$

\begin{tabular}{|c|c|c|c|}
\hline I) & Parameters of PCT & Cases(n=50) & Controls $(n=50)$ \\
\hline 1. & Sample size (n) & 50 & 50 \\
\hline 2. & Means & 51.36 & 0.03368 \\
\hline 3. & Standard deviation & 32.45 & 0.007003 \\
\hline 4. & Mean difference & \multicolumn{2}{|l|}{51.32} \\
\hline 5. & Standard Error & \multicolumn{2}{|c|}{4.58} \\
\hline 6. & Z - statistic & \multirow{2}{*}{\multicolumn{2}{|c|}{$\begin{array}{l}11.20 \\
P<0.001 \text { ( Highly significant) }\end{array}$}} \\
\hline & P value & & \\
\hline & \multicolumn{3}{|l|}{ Parameters of WBCs } \\
\hline \multicolumn{2}{|c|}{ 1. $\quad$ Means } & 24.70 & 7,508 \\
\hline \multicolumn{2}{|c|}{ 2. $\quad$ Standard deviation } & $10,998.26$ & 1152.60 \\
\hline & Mean difference & \multicolumn{2}{|l|}{16962.9} \\
\hline & Standard Error & \multicolumn{2}{|l|}{1563.90} \\
\hline & Z - statistic & \multicolumn{2}{|l|}{10.84} \\
\hline 6. & P value & \multicolumn{2}{|c|}{$\mathrm{P}<0.001$ (Highly significant) } \\
\hline III) & \multicolumn{3}{|c|}{ Correlation between WBC \& PCT Counts in Cases $\left(\mathbf{n}_{1}=\mathbf{5 0 , n _ { 2 } = 5 0 )}\right.$} \\
\hline 1. & \multicolumn{3}{|c|}{$\mathrm{r}$ - Value $=0.1578 ;$ nearer to plus 1 is positive correlation. } \\
\hline 2. & Significance test result & \multicolumn{2}{|c|}{$t=1.11$ with 98 d.f $; P .>0.05$ (Not significant ) } \\
\hline IV) & \multicolumn{3}{|c|}{ Correlation between WBC \& PCT Counts in Controls $\left(\mathbf{n}_{1}=\mathbf{5 0}, \mathbf{n}_{2}=50\right)$} \\
\hline 1. & \multicolumn{3}{|c|}{$r$ - Value $=0.9373$; nearer to plus 1 is positive correlation. } \\
\hline & Significance test result & \multicolumn{2}{|c|}{$\begin{array}{l}\mathrm{t}=18.51 ; \text { with } 98 \text { d.f } ; \mathrm{P}<0.001 \text { (Highly } \\
\text { significant) }\end{array}$} \\
\hline
\end{tabular}

Fig -1

COMIPARISON OF MEANS \& SD'S IN PCT

CATEGORI OF CASES \& CONTROLS 


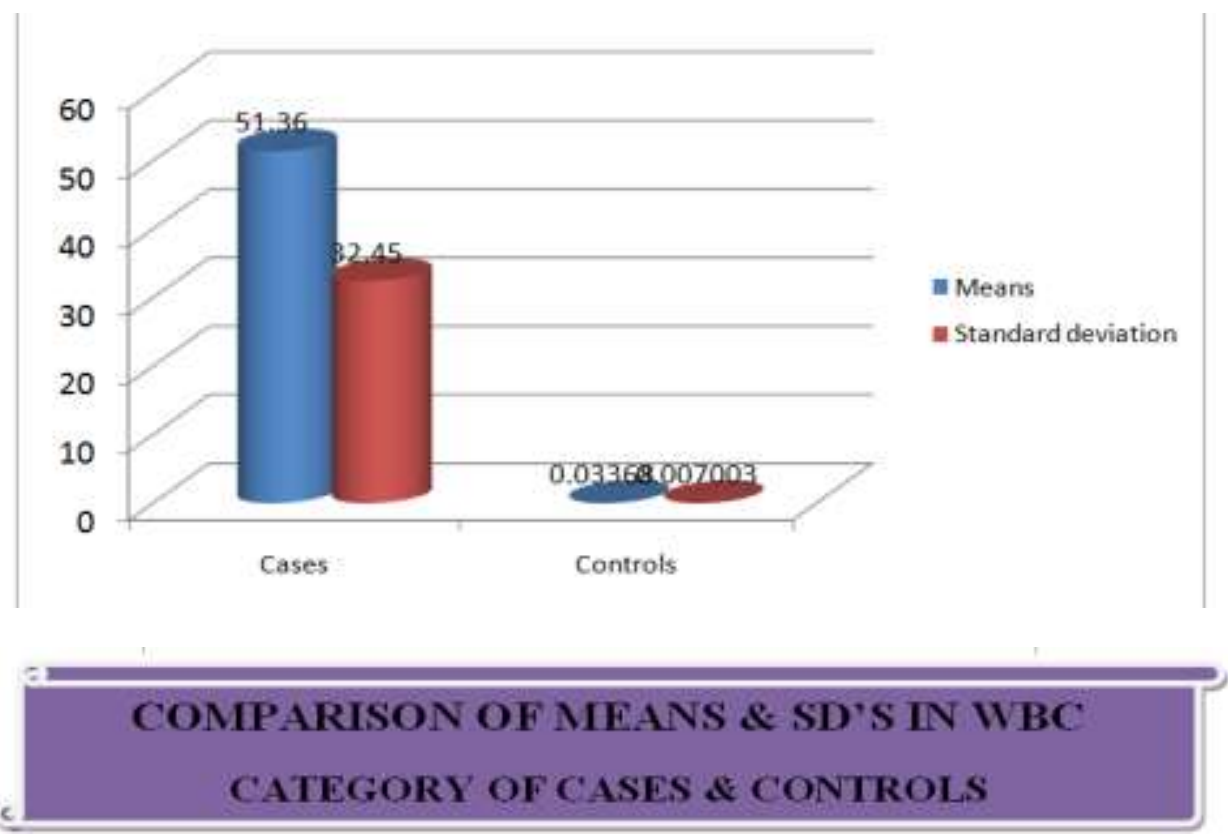

Fig -2

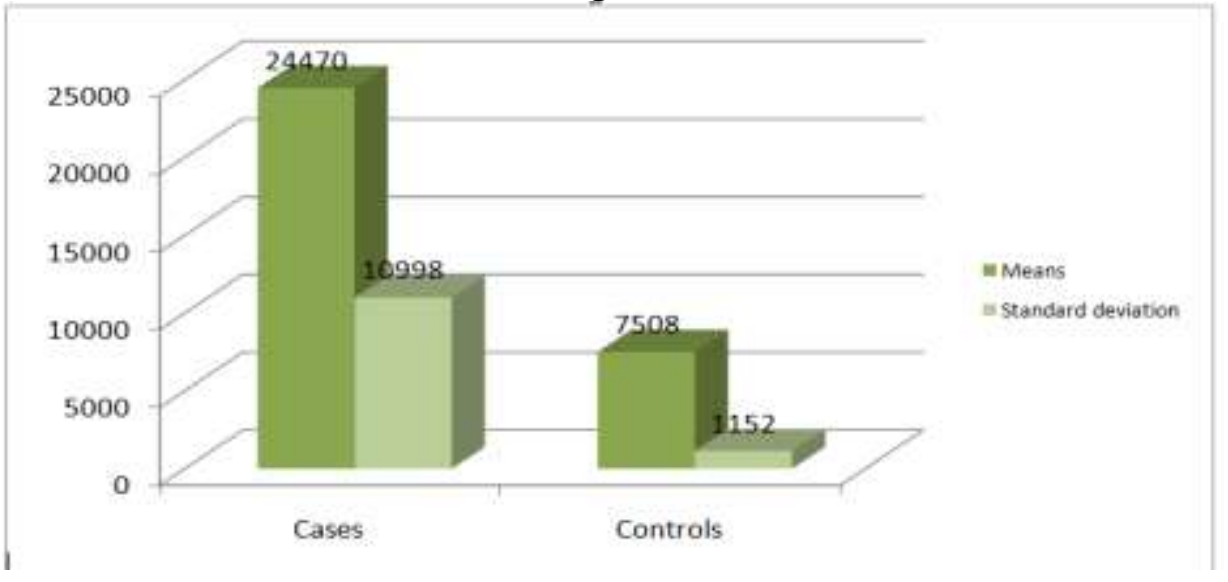

Fig-3

CORRELATION BETWEEN PCT \&WBC COUNTS IN CASES

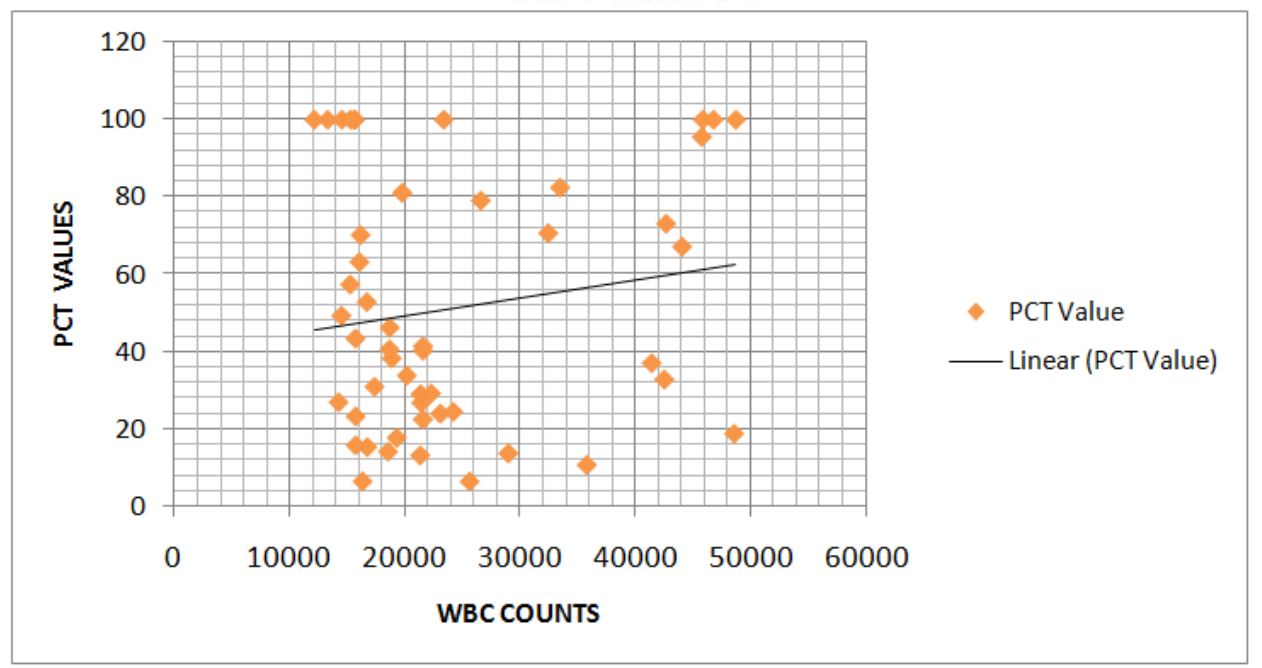




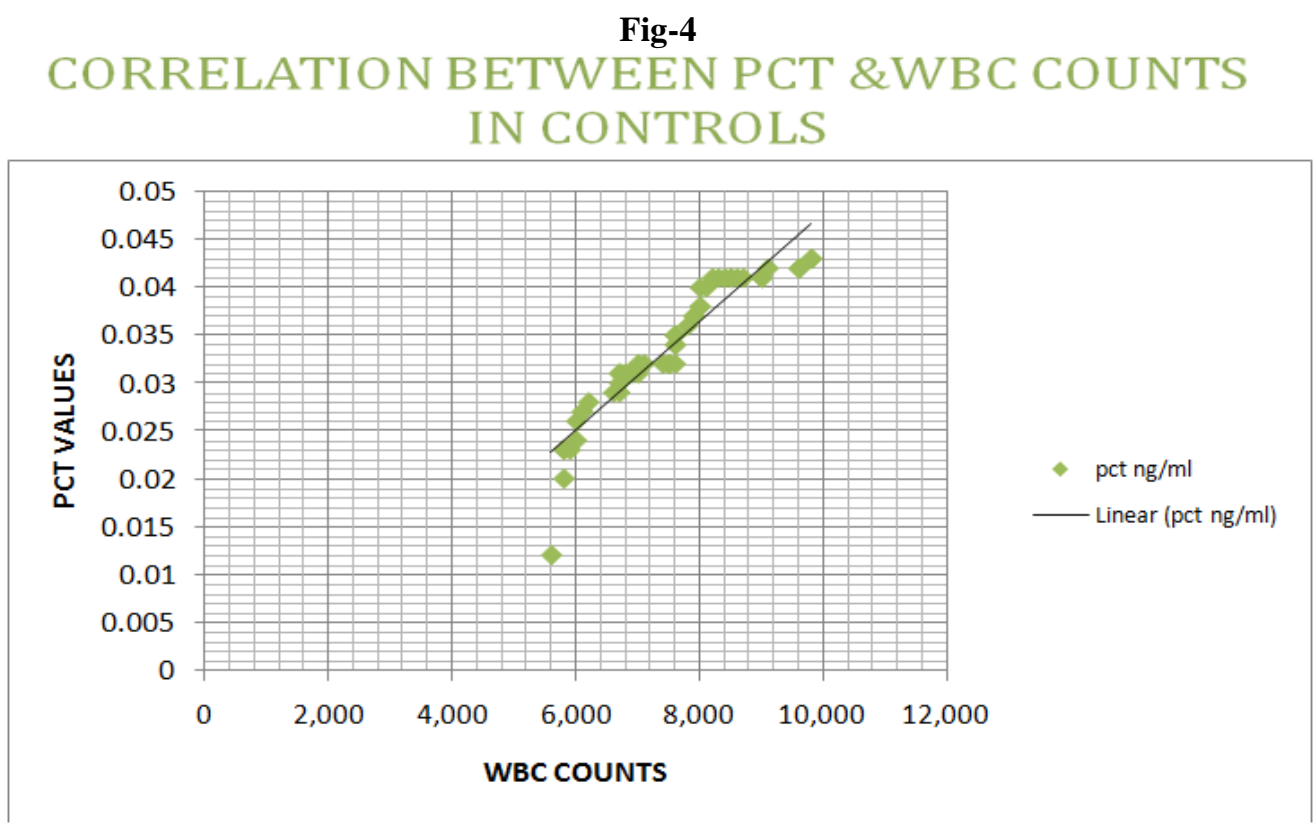

\section{Discussion}

In the present study we have taken 50 cases with sepsis and 50 controls. The mean value of PCT in cases is 51.36and controls is 0.3368.Standard deviation of PCT in cases is 32.45 and controls is 0.007.standard error is 4.58.p value is $<0.001$ which is highly significant.Serum PCT Levels have also been noted to increase with increasing severity of sepsis and organ dysfunction. This was demonstrated by giamarellos-bourboulis et al.(5)This has led to interest in using PCT as a prognostic indicator in critical care patients and a number of studies have now been performed.One of the largest studies was performed by Jensen et al.(6)They found increase of PCT in sepsis and very high results correlated with high risk of mortality. Studies done by C.G.Chivate et al.showed statistically significant correlation of sepsis with increase in PCT values.(7)

The mean value of wbc in cases is 24.70 and controls is 7,508.p value is $<0.001$ which is highly significant.There is positive correlation between PCT and WBC in sepsis.Studies done by magrini L et al showed PCT combined with WBC best diagnostic and prognostic power at ROC analysis in sepsis.(8)

Serum PCT, normally produced in the C-cells of the thyroid gland, is the precursor of calcitonin. A specific protease cleaves serum PCT to calcitonin, catacalcin, and an N-terminal residue. Normally, all serum PCT is cleaved and none is released into the blood stream. Serum PCT levels are therefore undetectable $(<0.1 \mathrm{ng} / \mathrm{ml}) \mathrm{in}$ healthy humans. During severe infections with systemic manifestations, however, serum PCT levels may increase to over $100 \mathrm{ng} / \mathrm{ml}$. In these conditions, serum PCT is probably produced by extra-thyroid tissues. Patients who have previously undergone total thyroidectomy still produce high levels of serum PCT during severe infection. The exact origin of serum PCT during sepsis is uncertain.

The (patho) physiological role of serum PCT during sepsis is not clear.(9,10) Serum PCT levels increase during severe generalized bacterial, parasitic or fungal infections with systemic manifestation. In severe viral infections, or inflammatory reactions of non-infectious origin, serum PCT levels do not increase or only show a moderate increase. Compared to the relatively short half-lives of cytokines such as tumor necrosis factor (TNF)-a and interleukin (IL)-6, the half-life of serum PCT in the systemic circulation is 25-30 hours rather long.(11) Because of these properties, serum PCT has been proposed as an indicator of severe generalized infections or sepsis $(12,13,14)$

Serum PCT is not a marker of infection as such since localized infections or infections with no systemic manifestation cause a limited, if any, increase in serum PCT levels. Although elevated serum PCT values during severe infections may decrease to very low levels with appropriate therapy, this does not always indicate complete eradication of the infection but only that generalization of the infection or the systemic response is under control. (15) Systemic inflammatory syndrome of non-infectious etiologies also leads to increases in serum PCT levels. Patients after major trauma or surgery and patients after cardiopulmonary bypass may present with increased serum PCT levels without any evidence of severe infection. However, the median values under these conditions are usually lesser than those found during severe sepsis and septic shock.(16) 


\section{Conclusion}

PCT alone or combined with WBC is a useful biomarker in early diagnosis of sepsis. Only procalcitonin improves accuracy of clinical sepsis diagnosis .PCT evaluation is a costly test but should be included in routine lab tests in sepsis patients for better patient outcome.

Conflict of interest: There is no conflict of interest to be declared.

Acknowledgment: I thank my husband and friends who helped me .

\section{References}

[1]. Robert S.Munford.Severe sepsis and septic shock.pg 2223,chap 271.Harrisons principles of internal medicine .18th edition.volLongo,fauci et al.2012.United states of America.

[2]. Mark A.Simmons.Autocoids and related drugs.pg 342,chapter 32.pharmacology an illustrated review.2012.Thieme medical and scientific publishers.India.

[3]. Schneider HG,Lam QT, procalcitonin for clinical laboratory:A review.Pathology.2007;39:383-390.

[4]. Assicot M,Gendrel D,Garsin H.et al.High serum procalcitonin concentrations in patients with sepsis and infection.Lancet.1993:341:515-518.

[5]. Giamarellos-Bouboulis EJ, Mega A,Grecka P et al.Procalcitonin:A marker to clearly differenciate systemic inflammatory response syndrome and sepsis in the critically ill patient?Intensive Care Med 2002;28:1351-6.

[6]. Jensen JU,Heslet L,Jensen TH et al.Procalcitonin increase in early identification of critically ill patients at high risk of mortality.Crit Care Med 2006;34:2596-602.

[7]. Chivate CG et al.Procalcitonin as a marker for diagnosis of sepsis.Int j res med sci.2016 Apr ;4(4):1216-1218.

[8]. Magrini L et al.Clin Chem. Lab Med.2014 oct;52(10):1465-72.doi:10.1515/cclm-2014-0210.

[9]. Meisner M. The prognostic value of PCT. In: Meisner M, editor. Procalcitonin (PCT)-A new, innovative infection parameter. Biochemical and clinical aspects. 3rd ed. New York: Thieme Publishers; 2000. p. 63-8

[10]. Becker KL, Nylén ES, White JC, Müller B, Snider RH Jr. Clinical review 167: Procalcitonin and the calcitonin gene family of peptides in inflammation, infection, and sepsis: a journey from calcitonin back to its precursors. J Clin Endocrinol Metab 2004;89:1512-25

[11]. Meisner M. Pathobiochemistry and clinical use of procalcitonin. Clin Chim Acta 2002;323:17-29.

[12]. Müller B, Becker KL, Schächinger H, Rickenbacher PR, Huber PR, Zimmerli W, et al. Calcitonin precursors are reliable markers of sepsis in a medical intensive care unit. Crit Care Med 2000;28:977-83

[13]. Chan YL, Tseng CP, Tsay PK, Chang SS, Chiu TF, Chen JC. Procalcitonin as a marker of bacterial infection in the emergency department: an observational study. Crit Care Med 2004;8:12-20.

[14]. Chan YL, Tseng CP, Tsay PK, Chang SS, Chiu TF, Chen JC. Procalcitonin as a marker of bacterial infection in the emergency department: an observational study. Crit Care Med 2004;8:12-20.

[15]. Meisner M, Reinhart K. Is procalcitonin really a marker of sepsis? Int J Intensive Care 2001;8:15-25.

[16]. Brunkhorst FM, Wegscheider K, Forycki ZF, Brunkhorst R. Procalcitonin for early diagnosis and differentiation of SIRS, sepsis, severe sepsis, and septic shock. Intensive Care Med 2000;26:148-52. 